\title{
Study on Drilling Parameters Optimization of Aluminum Alloy-Steel Hybrid Drill Pipe
}

\author{
Ni Hongjian, Tang Zhongmin * \\ School of Petroleum Engineering of China University of Petroleum, Qingdao, China \\ Email address: \\ nihj@upc.edu.cn (Ni Hongjian), 1350083618@qq.com (Tang Zhongmin) \\ ${ }^{*}$ Corresponding author
}

\section{To cite this article:}

Ni Hongjian, Tang Zhongmin. Study on Drilling Parameters Optimization of Aluminum Alloy-Steel Hybrid Drill Pipe. Science Discovery. Vol. 7, No. 6, 2019, pp. 436-440. doi: 10.11648/j.sd.20190706.20

Received: November 15, 2019; Accepted: December 9, 2019; Published: December 12, 2019

\begin{abstract}
With the increasing number of wells with complex operating conditions, the requirements for oil exploration equipment and technology are higher than ever before. Aluminum alloy drill pipe gradually receives good graces because of its light weight, high strength to mass ratio and strong corrosion resistance. This paper calculates different drillstring combinations according to the same horizontal well. It is found that the hook load and total friction resistance of the aluminum alloy-steel drillstring combination are smallest in the horizontal well, and aluminum alloy-steel drillstring combination is the optimal design. This paper studies the dynamic characteristics of aluminum alloy drill pipe by nonlinear finite element method. The simulation results show that rotation speed has a great influence on the trajectory of the aluminum alloy drill string in the vertical section. When the rotation speed is large, the centrifugal force makes the drill string move close to the wall, and the wear of the drill string is intensified. Besides, The moving range of the rotating drill string in the stable inclined section is close to the lower right part of the wellbore. When the WOB is constant, the range and amplitude of the lateral vibration of the drill string in the $\mathrm{X}$ and $\mathrm{Y}$ directions become larger as the rotational speed increases.
\end{abstract}

Keywords: Aluminum Alloy Drill Pipe, Horizontal Well, Motion Trail

\section{铝合金-钢混合型钻杆的钻井参数优化研究}

倪红坚, 唐仲旻*

中国石油大学 (华东) 石油工程学院, 青岛, 中国

邮箱

nihj@upc.edu.cn（倪红坚）, 1350083618@qq.com（唐仲旻）

摘要：随着复杂工况井的数量增多，作业现场对石油勘探设备与技术的要求变得更高。铝合金钻杆以其质量轻、强度 与质量比大、耐腐蚀性强等特点越来越受到钻井技术人员的青睐。本文根据所给水平井，计算不同钻柱组合后，发现 在水平井中铝合金-钢钻柱组合的大钩载荷与总摩阻较小。本文还借助非线性有限元法对铝合金钻杆进行动力学特性研 究, 模拟计算表明, 钻压增大对不同井段下钻柱的运动轨迹影响不大。而转速增加会使直井段钻柱紧贴井壁运动, 水 平段钻柱的横向振动加剧。

关键词: 铝合金钻杆, 水平井, 运动轨迹 


\section{1. 引言}

目前, 由于石油、天然气的消耗量日益增加, 油气 资源的勘探对象逐渐由常规油气转向非常规油气。由于 开发对象的转变, 超深井、水平井的数量必然会增多, 作业现场对石油勘探开发设备与技术的要求自然也更 高。普通钢质钻杆复杂工况井中常常发生摩擦热裂、应 力腐蚀断裂等事故, 影响钻井的速度和效率。而铝合金 钻杆由于其质量轻、强度与质量比大、耐腐蚀性强等特 点[1], 已经被广泛使用于俄罗斯的深井、定向井和大陆 架海洋钻井中 [2]。

我国对于铝合金钻杆的研究起步较晚 [3][4], 为了 应对愈加复杂的勘探开发井况, 必须认真研究铝合金钻 杆的相关特性。而目前国内对于铝合金钻杆的研究存在 很多局限性: 动力学特性方面的研究对象大多为直井或 斜直井 [5], 没有研究整个水平井的动力学特性; 而且, 到目前为止还没有针对现场实际井况的铝合金钻杆设 计方法的研究。复杂工况致使钻柱失效, 是钻井施工过 程中一个最为常见且昂贵的井内事故, 因此开展钻柱力 学研究具有很重要的意义。笔者使用钢钻柱、铝合金钻 柱和铝合金-钢复合钻柱对所给水平井进行钻柱组合设 计, 对比分析三种设计方案的优缺点。同时利用非线性 有限元方法, 对铝合金-钢钻柱组合的振动特性及浴动 特性进行分析, 从动力学角度解释钻杆容易形成划痕、 发生磨损、钻柱疲劳失效的原因, 根据理论研究结果为 合理选择钻井参数提供依据。

\section{2. 定向井铝合金钻杆设计方案研究}

根据所给水平井, 设计其钻柱组合。水平井分别用钢 质钻杆、纯铝合金钻杆、钢-铝合金复合钻杆进行设计, 并且通过比较大钩载荷和总摩阻, 选出最优方案 [6]。

\section{1. 水平井钻柱设计方法}

钻柱设计时应着重考虑为水平井段选择合适的钻柱 构件, 安全传递钻压的同时尽可能减小钻柱总重量。水平 井段较短时, 可以使用较重的管材来传递较高的轴向压力。 水平井段较长时, 应尽量采用轻质管材, 避免增大钻柱的 提升载荷和下行阻力[7]。

\subsection{1. 倾斜井眼钻柱失稳模型}

可根据Dowson公式计算处于倾斜圆柱孔内低边的圆 柱杆件不发生失稳屈曲的最大压缩载荷[8]:

$$
F_{\mathrm{c}}=2\left(\frac{E I q_{m} \sin \theta}{r}\right)^{\frac{1}{2}}
$$

水平段钻柱不发生失稳屈曲的最大压缩载荷可通过 吴疆 (Jiang Wu) 得出的公式计算:

$$
F_{c}=2\left(\frac{E I q_{m}}{r}\right)^{\frac{1}{2}}
$$

\subsection{2. 弯曲井段轴向力计算方法}

下图表示弯曲井段作用于钻柱单元上的力。其中正压 力 $\mathrm{N}$ 为:

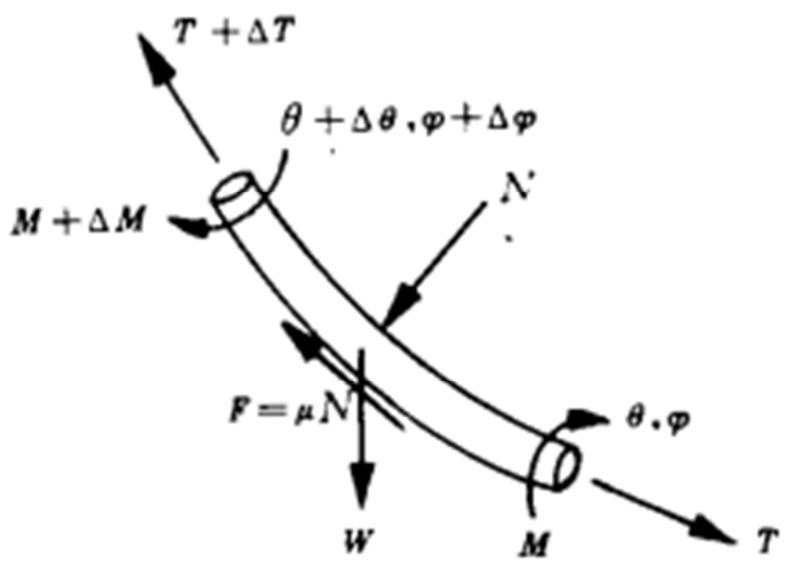

图1 钻进时作用在钻柱单元上的力。

$$
N=\left[(T \Delta \phi \sin \bar{\theta})^{2}+(T \Delta \theta+W \sin \bar{\theta})^{2}\right]^{\frac{1}{2}}
$$

轴向力和扭矩数学表达式为 [8]:

$$
\begin{aligned}
& T_{\mathrm{j}}=\mathrm{p}_{\text {头 }}+\sum_{i=1}^{j} \Delta T_{i} \\
& M_{\mathrm{j}}=\mathrm{M}_{\text {头 }}+\sum_{i=1}^{j} \Delta M_{i}
\end{aligned}
$$

为保证钻柱不失稳应满足以下条件:

$$
T_{\mathrm{j}} \leq F_{\mathrm{cj}}
$$

\section{2. 实例计算}

水平井基本参数如下:

直井段: $1490 \mathrm{~m}$;

造斜段：1490-2091.38m，造斜率 $4.7^{\circ} / 30 \mathrm{~m}$, 井径 $215.9 \mathrm{~mm}$;

水平段: $2091.38 \mathrm{~m}-5150 \mathrm{~m}$, 井径 $215.9 \mathrm{~mm}$;

环空油基钻井液密度取 $1.65 \mathrm{~g} / \mathrm{cm} 3$, 钻压取 $50 \mathrm{kN}, \mu$ 取 0.3 。

钢制钻杆

水平段设计：选取外径 $127 \mathrm{~mm}$, 内径 $108.6 \mathrm{~mm}$, 线重 $284.68 \mathrm{~N} / \mathrm{m}$, 钢级 $\mathrm{E}$ 的钻杆。钢材弹性模量取 $206 \mathrm{GPa}$, 普 通钻杆、加重钻杆惯性矩分别为, $5.94 \times 10^{-6} \mathrm{~m}^{4}, 11.11 \times 10^{-6} \mathrm{~m}^{4}$ 。 浮重: 普通钻杆取 $225 \mathrm{~N} / \mathrm{m}$, 加重钻杆取 $583 \mathrm{~N} / \mathrm{m}$ 。

$$
\begin{gathered}
F_{\mathrm{c}}=2 \sqrt{\frac{206 \times 10^{9} \times 5.94 \times 10^{-6} \times 284.68 \times 0.79}{0.04445}}=157.37 \mathrm{kN} \\
F_{\text {cchw }}=2 \sqrt{\frac{206 \times 10^{9} \times 11.11 \times 10^{-6} \times 738 \times 0.79}{0.0445}}=346.52 \mathrm{kN}
\end{gathered}
$$


经过计算可以发现, 使用普通钻杆在 $3440 \mathrm{~m}$ 时, 轴向 力为 $156.734 \mathrm{kN}$, 非常接近临界屈曲载荷, 为了避免发生 屈曲, 所以在此处应更换加重钻杆。

在造斜段 $1730 \mathrm{~m}$ 处, 受压载荷降低为 $145.309 \mathrm{kN}$, 此 时轴向力小于普通钻杆的临界屈曲载荷, 不必再使用加重 钻杆, 所以再次更换为普通钻杆。

(2) 铝合金钻杆

选取外径 $147 \mathrm{~mm}$, 内径 $121 \mathrm{mmD} 16 \mathrm{~T}$ ADP147×13铝合 金钻杆, 线重 $185.96 \mathrm{~N} / \mathrm{m}$, 铝合金钻杆弹性模量取 $72 \times 10^{9}$, 浮重取 $62.79 \mathrm{~N} / \mathrm{m}$ 。

取 $30 \mathrm{~m}$ 为一个单元, 通过计算单元的轴向力可知, 在 水平段 $2091.38 \mathrm{~m}$ 处该铝合金钻杆发生屈曲, 换用外径 $168 \mathrm{~mm}$, 内径 $146 \mathrm{mmD} 16 \mathrm{TADP} 168 \times 11$ 铝合金钻杆, 线重 $233.33 \mathrm{~N} / \mathrm{m}, \mathrm{KB}=0.41, \mathrm{I}=1.679 \times 10-5 \mathrm{~m} 4$, 浮重取 $62.25 \mathrm{~N} / \mathrm{m}$ 。

$$
F_{\mathrm{cc}}=2 \sqrt{\frac{72 \times 10^{9} \times 1.239 \times 10^{-5} \times 185.96 \times 0.41}{0.03445}}=88.866 \mathrm{kN}
$$

使用该钻杆至造斜段 $2030 \mathrm{~m}$ 处时, 受压载荷为 $86.254 \mathrm{kN}$, 可以更换为之前使用的铝合金钻杆, 一直使用 到井口处。

钢-铝合金复合钻杆

在 水平段选取外径 $147 \mathrm{~mm}$, 内径 $121 \mathrm{~mm}$ D16TADP147 × 13铝合金钻杆, 线重 $185.96 \mathrm{~N} / \mathrm{m}$, 弹性模量 取 $72 \times 10^{9}$, 惯性矩取 $\mathrm{I}=1.239 \times 10^{-5} \mathrm{~m}^{4}, \mathrm{~KB}=0.41$, 浮重取 $62.79 \mathrm{~N} / \mathrm{m}$ 。

$$
F_{\mathrm{cc}}=2 \sqrt{\frac{72 \times 10^{9} \times 1.239 \times 10^{-5} \times 185.96 \times 0.41}{0.03445}}=88.866 \mathrm{kN}
$$

经过计算可知, 在水平段 $2091.38 \mathrm{~m}$ 处该铝合金钻杆会 发生屈曲。故在 $2120 \mathrm{~m}$ 处换用外径 $127 \mathrm{~mm}$, 内径 $108.6 \mathrm{~mm}$, 线重 $284.68 \mathrm{~N} / \mathrm{m}$, 钢级 $\mathrm{E}$ 的钻杆。钢材弹性模量取 $206 \mathrm{GPa}$ 普通钻杆惯性矩取 $5.94 \times 10^{-6} \mathrm{~m}^{4}$ 。

$$
F_{\mathrm{cc}}=157.37 \mathrm{kN}
$$

在 $2000 \mathrm{~m}$ 处, 单元轴向力降低到 $88.091 \mathrm{kN}$, 可以更换 为之前使用的铝合金钻杆。

\section{3. 对比分析三种钻柱组合}

大钩载荷是选择钻机的重要依据, 大钩载荷越小, 所 选钻机的型号也越小, 成本自然也越低。钻柱屈曲和摩阻 会影响钻柱轴向力的传递, 导致钻头无法获得有效的钻压。 在这里, 我们对比了三种钻柱组合下的大钩载荷和总摩阻, 从而选择更优的钻柱组合类型。

\section{表1 大钩载荷和总摩阻。}

\begin{tabular}{lll}
\hline 钻柱组合类型 & 大钧载荷 $/ \mathbf{k N}$ & 总摩阻 $/ \mathbf{k N}$ \\
\hline 钢质钻杆 & 261.314 & 352.647 \\
铝合金钻杆 & 54.054 & 81.537 \\
钢-铝合金钻杆 & 51.888 & 88.561 \\
\hline
\end{tabular}

从上表的数据不难看出, 纯铝合金钻杆组成的钻柱和 钢-铝合金复合钻杆组成钻柱的大钩载荷和总摩阻都是比 较理想的, 二者相差不大。但是, 纯铝合金钻柱组合使用 了两种尺寸相差较大的钻杆, 没有缓和二者刚度的变化。 而且从成本方面考虑, 纯铝合金钻杆在水平段 $2091.38 \mathrm{~m}$ 处 发生屈曲, 换用更高强度的D16T ADP168×11铝合金钻杆, 这就一定程度上增加了成本。而钢-铝合金钻杆在 $2120 \mathrm{~m}$ 处 换用了外径 $127 \mathrm{~mm}$, 内径 $108.6 \mathrm{~mm}$ 钢级 $\mathrm{E}$ 的钻杆。所以钢铝合金复合钻杆在一定程度上节约了成本。

综上所述, 在该水平井的钻柱组合设计中, 铝合金钢钻柱组合是最优设计方案。

\section{3. 铝合金钻杆的动力学特性研究}

\section{1. 钻柱动力学有限元模型}

\subsection{1. 基本假设}

本章分析钻柱动力学特性, 采用如下假设: (1) 忽略 剪切应变; (2) 井眼截面为圆形; (3) 忽略钻柱接头的影 响[9]。

\subsection{2. 边界条件}

钻柱两端的边界条件: 上端位于井口处, 铰支在井眼 中心, 受到拉力和扭矩作用; 下端是钻头, 钻头受到钻压 和扭矩作用, 是球铰边界 $[10,11]$ 。

\subsection{3. 钻柱动力学的直梁单元模型}

钻柱动力学特性的计算涉及到几何非线性、接触非线 性的问题。钻柱外径的变化以及井眼的不规则, 会导致钻 柱与井壁之间的间隙不同。实际现场作业时, 在地面设备 的驱动下, 钻柱与井壁不断接触碰撞, 并且接触碰撞的位 置也在变化, 所以钻柱的运动实际上结合了自转和公转, 集中了横向振动、纵向振动、扭转振动和浴动 $[12,13]$ 。

钻柱系统动力学平衡方程为 [14]:

$$
[M]\{\ddot{\mathrm{U}}\}+[\mathrm{C}]\{\dot{U}\}+[K]\{U\}=\{F\}
$$

\section{2. 数值模拟结果分析与讨论}

本文主要针对利用铝合金钻杆钻水平井的过程中, 复 合钻进时铝合金钻杆的动力学特性进行分析, 通过研究铝 合金钻杆的运动形式 (运动轨迹、速度等), 判断钻杆是 否容易发生磨损, 从而为优选钻井参数 (钻压、转速) 提 供理论依据 $[15,16]$ 。

\subsection{1. 直井段铝合金钻杆的动力学特性分析}

所用钻具组合: $\Phi 311.2 \mathrm{~mm}$ 钻头 $+\Phi 241.3 \mathrm{~mm}$ 单弯螺杆 + 钻具止回 $+\Phi 203.2 \mathrm{~mm}$ 无磁钻铤 $\times 1$ 根 $+\Phi 203.2 \mathrm{~mm}$ 钻铤 $\times 4$ 根 $+\Phi 203.2 \mathrm{~mm}$ 随钻震击 $+\varphi 203.2 \mathrm{~mm}$ 钻铤 1 根 $+\varphi 178.8 \mathrm{~mm}$ 钻铤 3 根 $+\Phi 168 \mathrm{mmD} 16 \mathrm{TADP} 168 \times 11$ 铝合金钻杆 20 根 $+\Phi 147 \mathrm{mmD} 16 \mathrm{~T}$ ADP $147 \times 13$ 铝合金钻杆 + 下旋塞 2 个+方钻 杆+上旋塞。

转速对钻杆运动特性的影响 
下图分别是 $10 \times 10^{4} \mathrm{~N}$ 钻压下, $30 \mathrm{RPM} 、 60 \mathrm{RPM}$ 和 90RPM转速下, 井深2700米处的钻柱中心运动轨迹图。
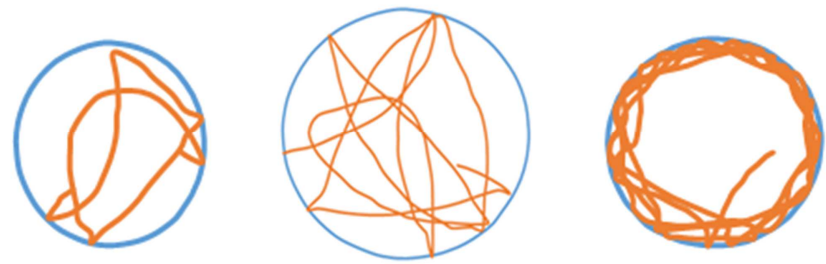

图2 30、60和90RPM转速下, 井深2700m处的钻柱中心运动轨迹图。

从图 2 可以看出, 转速对钻柱的运动轨迹有很大的影 响, 转速较小时钻柱和井壁不连续接触, 不会发生紧贴井 壁的旋转运动; 当转速较大时, 离心力较大, 钻柱开始贴 紧井壁运动, 钻柱的磨损加剧, 60RPM时钻柱贴井壁公转 的平均角速度为 $37 \mathrm{RPM}$, 继续增大转速至 $90 \mathrm{RPM}$ 时, 浴动 加剧。

因此，对该铝合金钻柱而言，应该控制其转速，经模 拟表明, 钻柱转速小于 51 RPM 时钻柱不会与井壁持续涡动, 有利于延长钻柱的使用寿命。

钻压对运动特性的影响

下图是转速为60RPM时不同钻压下, 井深2700米处的 钻柱中心运动轨迹图。
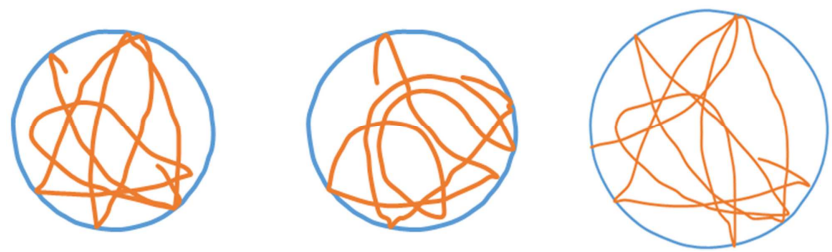

图3 $6 \times 10^{4} \mathrm{~N} 、 8 \times 10^{4} \mathrm{~N}$ 和 $10 \times 10^{4} \mathrm{~N}$ 钻压下, 井深 2700 米处的钻柱中心运动 轨迹图。

从图3可以看出, 钻压 $\left(6 \sim 8 \times 10^{4} \mathrm{~N}\right.$ 范围内 $)$ 对钻柱的 运动轨迹影响不大, 相对较大的钻压不会改变该铝合金钻 柱组合的运动特性。

\subsection{2. 稳斜段铝合金钻杆的动力学特性分析}

所用钻具组合: $\Phi 311.2 \mathrm{~mm}$ 钻头 $+\Phi 244 \mathrm{~mm}(\Phi 197 \mathrm{~mm})$ $1.25^{\circ} \sim 1.75^{\circ}$ 单弯动力钻具 + 钻具止回阀 $+\Phi 203.2 \mathrm{~mm}$ 无磁 钻铤 $9 \mathrm{~m}+\mathrm{MWD}(\mathrm{LWD})$ 短节 $+\Phi 203.2 \mathrm{~mm}$ 无磁钻铤 $9 \mathrm{~m}$ $+\Phi 203.2 \mathrm{~mm}$ 钻铤 $\times 2$ 根 $+\Phi 158.8 \mathrm{~mm}$ 钻铤 $\times 9$ 根 + 旁通阀 + $\Phi 168 \mathrm{mmD} 16 \mathrm{~T}$ ADP $168 \times 11$ 铝合金钻杆 $\times 15$ 根 $+\Phi 158.8 \mathrm{~mm}$ 随钻震击器 $+\Phi 168 \mathrm{mmD} 16 \mathrm{~T}$ ADP $168 \times 11$ 铝合金钻杆 $\times 15$ 根 $+\Phi 147 \mathrm{~mm}$ D16T ADP $147 \times 13$ 铝合金钻杆 + 下旋塞 2 个+方 钻杆+上旋塞。

转速对钻杆运动特性的影响

稳斜段同样使用 $30 \mathrm{RPM}$ 、60RPM和 $90 \mathrm{RPM}$ 的转速来 模拟钻杆的运动轨迹。下图分别是保持 $6 \times 10^{4} \mathrm{~N}$ 钻压, 30RPM、60RPM和 $90 \mathrm{RPM}$ 转速下, 井深3800米处的钻柱 中心运动轨迹图。
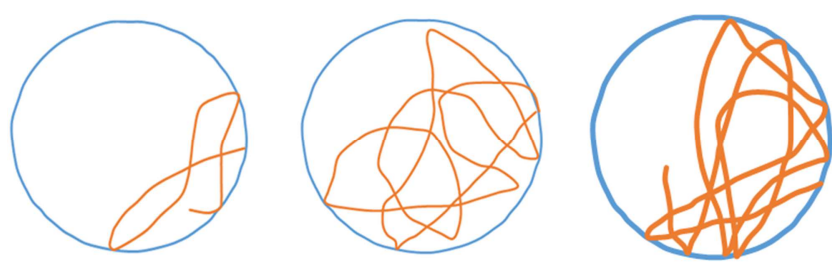

图4 30、60和90RPM转速下, 井深3800m处的钻柱中心运动轨迹图。

由图4可以看出, 稳斜段旋转钻柱的运动范围逐渐靠 近井筒的右下部。当钻压一定的情况下, 随着转速的增加, 钻柱的横向振动的范围和幅度越来越大, $\mathrm{X} 、 \mathrm{Y}$ 两个方向 的横向振动愈加剧烈。

钻压对运动特性的影响
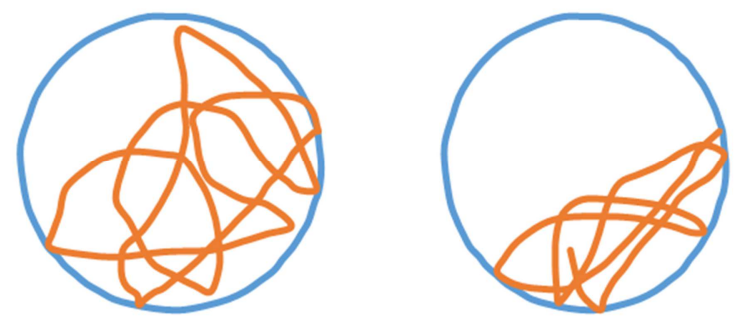

图5 6 $\times 10^{4}$ 和 $10 \times 10^{4} \mathrm{~N}$ 钻压下, 井深 3800 米处的钻柱中心运动轨迹图。

从图 5 可以看到, 在两个不同钻压下, 铝合金旋转钻 柱中心的运动轨迹曲线变化较小, 但是运动范围变大。随 着钻压的增大, 铝合金钻柱的运动轨迹由最初近似圆形逐 渐被压扁。但从整体上看, 钻压对其钻柱中心的运动轨迹 影响并不大。

\subsection{3. 水平段铝合金钻杆的动力学特性分析}

所用钻柱组合: $\varphi 215.9 \mathrm{~mm}$ PDC钻头 $+\varphi 165 \mathrm{~mm}\left(0.75^{\circ}\right)$ 单弯螺杆钻具 + 钻具止回阀 $+\varphi 210 \mathrm{~mm}$ 扶正器 $+\varphi 127 \mathrm{~mm}$ 无 磁加重钻杆 + LWD+无磁短节 $+\Phi 168 \mathrm{mmD} 16 \mathrm{~T}$ ADP168×11 铝合金钻杆 6 根 $+\Phi 147 \mathrm{mmD} 16 \mathrm{~T}$ ADP $147 \times 13$ 铝合金钻杆 $2000 \mathrm{~m}+\Phi 168 \mathrm{mmD} 16 \mathrm{~T}$ ADP $168 \times 11$ 铝合金钻杆 21 根 + 旁通 阀 1 只 $+\Phi 127 \mathrm{~mm}$ 斜坡钻杆+旋塞 2 个。

转速对钻杆运动特性的影响

水平段使用 $30 \mathrm{RPM} 、 60 \mathrm{RPM}$ 和 $90 \mathrm{RPM}$ 的转速来模拟 钻杆的运动轨迹。下图分别是保持钻压 $6 \times 10^{4} \mathrm{~N}$, 转速 30RPM、60RPM和90RPM下, 井深4900米处的钻柱中心 运动轨迹放大图。
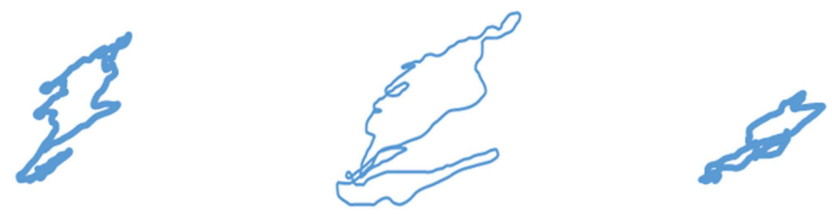

图6 30、60和90RPM转速下, 井深 $4900 \mathrm{~m}$ 处的钻柱中心运动轨迹图。

从上图可以看出, 水平段钻柱中心始终在井筒的右下 部运动。随着转速的增大, 钻柱横向振动的范围和幅度越 来越大, 这与稳斜段的变化规律类似。但是相较于稳斜段, 水平段旋转钻柱在Y方向的横向振动幅度没有稳斜段那么 大。 


\section{钻压对运动特性的影响}

由于水平段钻进使用的是PDC钻头, 所以钻压一般都 较小, 而且从稳斜段已经可以看到钻压对钻柱中心运动轨 迹影响并不大，所以这里不考虑钻压的影响。

\section{4. 结论}

本文使用钢钻柱、铝合金钻柱和铝合金-钢复合钻柱 对所给直井和水平井进行钻柱组合设计, 对比分析三种设 计方案的优缺点。同时利用非线性有限元方法, 对铝合金 -钢钻柱的运动轨迹进行分析, 从动力学角度解释钻杆容 易形成划痕、发生磨损的原因, 可得结论如下:

在水平井的设计中, 通过对比三种钻柱组合下的大钩 载荷和总摩阻可以看出: 铝合金钻柱和铝合金-钢钻柱的 大钩载荷远小于钢钻柱, 体现了铝合金钻杆具有密度小, 同等钻井液密度下浮力系数小的特性。至于总摩阻方面, 铝合金钻杆和钢-铝合金钻杆的总摩阻也远小于钢质钻杆 的总摩阻, 这与铝合金钻杆具有更小的弹性模量有密不可 分的关系。除此以外, 铝合金-钢钻柱考虑到了更换钻杆 时钻杆截面的刚度变化, 所以在该设计中铝合金-钢钻柱 组合是最优设计方案。

模拟计算表明: 在直井段, 转速对铝合金钻柱的运动 轨迹有很大的影响。转速较小时钻柱不发生紧贴井壁的旋 转运动, 当转速较大时, 离心力较大, 钻柱开始贴紧井壁 运动, 钻柱的磨损加剧。因此, 对该铝合金钻柱而言, 应 该控制其转速小51RPM, 有利于延长钻柱的使用寿命。直 井段钻压的变化对钻柱的运动轨迹影响不大。

在稳斜段, 旋转钻柱的运动范围逐渐靠近井筒的右下 部, 且随着转速的增加, 钻柱 $\mathrm{X} 、 \mathrm{Y}$ 两个方向的横向振动 愈加剧烈。而转速不变, 钻压增大时, 铝合金旋转钻柱中 心的运动轨迹曲线变化较小, 但是运动范围变大。最后, 在水平段钻柱中心始终在井筒的右下部运动。随着转速的 增大, 钻柱横向振动的范围和幅度越来越大, 这与稳斜段 的变化规律类似。但是相较于稳斜段, 水平段旋转钻柱在 Y方向的横向振动幅度较小。

\section{参考文献}

[1] 漂泰宁,薛维,兰凯. 高可靠性铝合金钻杆及其在超深井和水 平井中的应用 [J].地质科技情报,2010,(01):112-115.
[2]吕拴录,骆发前,周杰,刘远扬,苏建文,卢强.铝合金钻杆在塔 里木油田推广应用前景分析 [J]. 石油钻探技 术,2009,(03):74-77.

[3] 阎美萍.俄罗斯铝合金钻杆技术应用及前景分析[D].中国石 油大学 (华东) ,2013:3-6.

[4] 余荣华, 袁鹏斌. 铝合金钻杆的特点及应用前景[J].石油矿 场机械,2011,40(3):81-82.

[5] 杨尚谕. 基于深井/超深井的铝合金钻杆设计研究[A].中国 石油集团石油管工程技术研究院(TGRI)、美国石油学会 (API)、中国石油学会石油管材专业委员会、石油管材及装 备材料服役行为与结构安全国家重点实验室.油气井管柱 与管材国际会议 (2016) 论文集[C].中国石油集团石油管工 程技术研究院(TGRI)、美国石油学会(API)、中国石油学会 石油管材专业委员会、石油管材及装备材料服役行为与结 构安全国家重点实验室:,2016:5.

[6] 陈廷根, 管志川.钻井工程理论与技术 $[\mathrm{M}]$.东营: 中国石油 大学出版社, 2000.6:45-47.

[7] 韩志勇.水平井钻柱的优化设计问题 [J]. 石油大学学报(自然 科学版),1997,(05):24-26+114-115.

[8] 赵洪激,彭高华. 水平井钻柱设计方法 [J]. 天然气工 业, 1995,(06):35-39+100.

[9] 唐继平,狄勤丰,胡以宝,王文昌,梁红军,杨成新. 铝合金钻杆 的动态特性及磨损机理分析 [J]. 石油学 报,2010,(04):684-688.

[10] Khulicf Y A, Al-Sulaiman F A, Bashmal S. Vibration analysis of drillstrings with self-excited stick-slip oscillations [J]. Journal of Sound and Vibration, 2007: 540-558.

[11] 于永南.钻柱力学分析的有限元法[M].于永南, 石油大学出 版社,1998:10-13.

[12] Dykstra M W. Nonlinear drill string dynamics [D]. Ohio: The University of Tusla, 1996: 1-2.

[13] 胡以宝. 基于实际井眼轨迹的钻柱动力学特性有限元分析 [D].上海大学, 2011:20-23.

[14] Hakimi H, Moradi S. Drillstring vibration analysis using differential quadrature method $[\mathrm{J}]$. Journal of Petroleum Science and Engineering, 2010, 70 (3): 235-242.

[15] 署恒木, 吕英民, 蔡强康.下部钻具组合的动态分析 [J].石油 学报,1992,13(1):108-117.

[16] Klaus-Jürgen B. Finite element procedures in engineering analysis [M]. New Jersey: Prentice-Hall, Inc., 1982: 371-604. 